\title{
Exact reliability formula for a linear consecutive k-out-of-n: F system and relayed consecutive systems with a change point for any $k \leq n$, with stress-strength application
}

\author{
S.M.Bakry \\ Department of Basic Science \\ Faculty of Computer and Information Science, Ain Shams University, Cairo, Abbasia, Egypt \\ soaad_mohamed@cis.asu.edu.eg \\ N.A. Mokhlis \\ Department of Mathematics \\ Faculty of Science, Ain Shams University, Cairo, Abbasia, Egypt \\ nahedmokhlis@hotmail.com
}

\begin{abstract}
This paper presents exact formulas for the reliability of linear consecutive k-out-of-n: $\mathrm{F}$, and relayed consecutive k-out-of-n: $\mathrm{F}$ systems, having a change point at position $c, 1 \leq c \leq n$, for any $k \leq n$. A change point at position $c$, means that the components after this point have reliabilities that are different from those before or at position $c$. The components are assumed to be independent. Practically, the change in the components reliabilities may be due to change in the stress applied. Assuming a change in stress, exact formulas of the stress-strength reliability of the systems are derived, considering two cases. The first case assumed strength and stress having the same form of distributions, while the second case assumed strength and stress having different forms of distributions. Estimation of the stress-strength reliability for both cases is discussed. Applications to both cases are considered with numerical illustration.
\end{abstract}

Keywords: Linear (relayed linear) consecutive k-out-of-n: F system; Stress-strength reliability; General exponential form distribution; Generalized Lindley distribution, Maximum likelihood estimator.

\section{Notations}

$n \quad$ Number of components of the system.

$k \quad$ Minimum number of consecutive failed components required for system failure.

$N(j, s, r) \quad$ The number of ways in which $j$ identical balls can be placed in $s$ distinct urns subject to the requirement that at most $r$ balls are placed in any one urn.

$c \quad$ Position of the change point of the system, $1 \leq c \leq n$.

$\underline{\mathrm{P}} \quad$ Is a vector $\left[p_{1}, p_{2}\right]$.

$L / k / n / c \quad$ Linear consecutive k-out-of-n: F system with a change point at position $c$.

$L / k / n / c ; \underline{\mathrm{P}} \quad L / k / n / c$ with reliability $p_{1}$ for components from 1 to $c$, and reliability $p_{2}$ from $c+1$ to $n$.

$\mathrm{R}_{\mathrm{w}}(k, n, c ; \underline{\mathrm{P}})$ Reliability of a $L / k / n / c$; $\underline{\mathrm{P}}$, given that the component at position $c$ is working.

$\mathrm{R}_{F}(k, n, c ; \underline{\mathrm{P}}) \quad$ Reliability of a $L / k / n / c ; \underline{\mathrm{P}}$, given that the component at position $c$ is failed.

$\mathrm{R}(k, n, c ; \underline{\mathrm{P}}) \quad$ Reliability of a $L / k / n / c ; \underline{\mathrm{P}}$. 


$$
\begin{array}{cl}
\mathrm{R}_{u}(k, n, c ; \underline{\mathrm{P}}) & \text { Reliability of a relayed-unipolar } L / k / n / c ; \underline{\mathrm{P}} . \\
\mathrm{R}_{b}(k, n, c ; \underline{\mathrm{P}}) & \text { Reliability of a relayed-bipolar } L / k / n / c ; \underline{\mathrm{P}} . \\
\mathrm{R}_{(s: s)}(k, n, c) & \text { Stress-strength reliability of a } L / k / n / c . \\
\mathrm{R}_{(s: s) u}(k, n, c) & \text { Stress-strength reliability of a relayed-unipolar } L / k / n / c . \\
\mathrm{R}_{(s: s) b}(k, n, c) & \text { Stress-strength reliability of a relayed-bipolar } L / k / n / c . \\
\mathrm{X}, \text { and } \bullet & \text { Stands for a failed, and working component, respectively. }
\end{array}
$$

\section{Introduction}

A linear consecutive k-out-of-n: F system consists of $n$ components arranged linearly, the system fails if and only if $k$ or more consecutive components fail. There exist many practical applications of this type of systems, for example, the telecommunications system with $n$ relay stations, and the oil pipeline system with $n$ pump stations, given by Chiang and Niu (1981). Many researches in the literature concerned such systems. Derman et al. (1982) presented a formula for the reliability of a linear consecutive k-outof-n: F system with identical and independent components with reliabilities $p$, given by

$$
\mathrm{R}(k, n ; p)=\sum_{j=0}^{n} N(j, n-j+1, k-1) q^{j} p^{n-j}
$$

Lambiris and Papastavridis (1985) derived an expression for the numbers $N(j, n-j+1 ; k-1)$ in (1), with $n-j+1 \geq 0$, as follows

$$
N(j, n-j+1, k-1)=\sum_{\lambda=0}^{n-j+1}\left(\begin{array}{c}
n-j+1 \\
\lambda
\end{array}\right)\left(\begin{array}{c}
n-\lambda k \\
j-\lambda k
\end{array}\right)(-1)^{\lambda} \text {. }
$$

Other different formulas for the reliability of a linear consecutive k-out-of-n: F system are obtained by different methods, see Chiang and Niu (1981), Hwang (1982), Kossow and Preuss (1989), Ge and Wang (1990), Jung and Kim (1993), Chao et al. (1995), Mokhlis (2001), and Gökdere et al. (2016).

Also, relayed linear consecutive k-out-of-n: F systems are of practical importance. There are two types of relayed linear consecutive k-out-of-n: F systems: The first type is a relayed-unipolar linear consecutive k-out-of-n: F system which is a system consisting of $n$ components arranged in a line such that the system fails if the first component fails or at least $k$ consecutive components fail; while the second type is a relayed-bipolar linear consecutive k-out-of-n: F system, where the $n$ components are arranged in a line such that the system fails if the first or last component fails, or at least $k$ consecutive components fail. The notation of relayed-unipolar and bipolar consecutive k out-of-n: $F$ systems is given by Hwang (1988).

In the reliability theory, stress-strength reliability of a system with random strength $Y$ under the random stress $X$, is the probability that the strength exceeds the stress. Stressstrength analysis was first considered by Birnbaum (1956) and developed by Birnbaum and McCarty (1958). Johnson (1988) presented stress-strength models for the system reliability. To the authors' knowledge few researches have considered the stress-strength reliability problem of consecutive k-out-of-n systems. Ery1lmaz (2008) presented the 
multi component stress-strength reliability of consecutive k-out-of-n: G system, for both cases in which there is a change and no change in strength. Zhao et al. (2018) studied a two-stage shock model with self-healing mechanism. A change point is introduced to describe the two-stage failure processes of the system. They proposed three preventive maintenance policies for the system under different monitoring conditions.

Akici (2010) discussed a linear consecutive k-out-of-n: F system with a change point, using the longest run statistic under the condition of special values of $k, 2 k \geq n$. Akici (2010) mentioned a practical example of this model, which is the gas pipeline from Russia to Turkey. This pipeline system is under two different stresses $X_{1}$ (water) and $X_{2}$ (soil). The pipeline starts from Russia passes across the Black Sea, enter Turkey from Samsun, and ends in Ankara, in Samsun it leaves the sea and enters soil, which is the change point of this system, and there are many other practical examples. Akici (2010) discussed this situation for special values of $k$ satisfying $2 k \geq n$. However, in practice $k$ could take any value between 1 and $n$, not necessarily, $2 k \geq n$. So, in this paper we study the reliability of a linear consecutive k-out-of-n: F system with a change point at position $c$, when $k$ could take any possible value i.e., $1 \leq k \leq n$. Also, the reliabilities of the relayed-unipolar and relayed-bipolar linear consecutive k-out-of-n: F systems with a change point at position $c$ are derived for any $k, 1 \leq k \leq n$. Exact formulas for the linear and relayed linear (unipolar and bipolar) consecutive k-out-of-n: F systems are derived conditioning on the state (working or failed) of the component at position $c$, for any values of $k, 1 \leq k \leq n$. Formulas for $\mathrm{R}_{(s: s)}(k, n, c), \mathrm{R}_{(s: s) u}(k, n, c)$, and $\mathrm{R}_{(s: s) b}(k, n, c)$ are derived, for any distributions of strength of components $F(x)$ and stresses $G_{i}(x)$, $i=1,2$. As application, we discussed two cases: Case I and Case II. Case I, when $F(x)$ and $G_{i}(x), i=1,2$ have the same form (general exponential form), in this case exact formulas of $\mathrm{R}_{(s: s)}(k, n, c), \mathrm{R}_{(s: s) u}(k, n, c)$, and $\mathrm{R}_{(s: s) b}(k, n, c)$ are obtained. For illustrating these results numerically, the negative exponential distribution is taken as an example of the general exponential form. Case II, when $F(x)$ and $G_{i}(x), i=1,2$ have different forms (generalized Lindley distribution for strength and negative exponential distribution for stresses). The Generalized Lindley distribution includes as special cases the exponential, gamma and Lindley distributions, and it is used in stress-strength reliability modeling and analyzing lifetime data, see Elbatal et al. (2013).

The paper is organized as follows: In Section 2, we obtain explicit forms for $\mathrm{R}(k, n, c ; \underline{\mathrm{P}}), \mathrm{R}_{u}(k, n, c ; \underline{\mathrm{P}})$, and $\mathrm{R}_{b}(k, n, c ; \underline{\mathrm{P}})$. In Section 3 , we present the stressstrength reliability of the systems generally for any continuous distributions, assuming the change of components reliabilities is due to change in the stress applied. In Section 4, we obtain exact formulas for $\mathrm{R}_{(s: s)}(k, n, c), \mathrm{R}_{(s: s) u}(k, n, c)$, and $\mathrm{R}_{(s: s) b}(k, n, c)$ for case $\mathrm{I}$, and case II. In Section 5, the maximum likelihood estimators of the stress-strength reliabilities are obtained for both cases I \& II. In Section 6, a numerical illustration of the theoretical results is presented, to show the effect of $k, c$, and the parameters of the distributions on the reliabilities. Also, the performance of the estimators is detected. In Section 7, a conclusion is presented. 


\section{Reliability Formulas}

Assume we have a linear consecutive k-out-of-n: F system with independent components, having a change point at position $c$. This means that the first $c$ components are identical with reliability $p_{1}\left(q_{1}=1-p_{1}\right)$, while the remaining $(n-c)$ components are also identical but with a different reliability $p_{2}\left(q_{2}=1-p_{2}\right)$. The reliability of this system, $\mathrm{R}(k, n, c ; \underline{\mathrm{P}})$, is given by the following theorem.

\section{Theorem 1}

Assuming independent components, for any $k \leq n$, and $1 \leq c \leq n$, we have

$$
\begin{aligned}
& \mathrm{R}(k, n, c ; \underline{\mathrm{P}}) \\
& =\sum_{m=0}^{c-1} N(m, c-m, k-1) q_{1}^{m} p_{1}^{c-m} \\
& \times \sum_{j=m}^{n-c+m} N(j-m, n-c-j+m+1, k-1) q_{2}^{j-m} p_{2}^{n-c-j+m} \\
& +\sum_{r=0}^{k-2} \sum_{k=r}^{c-2} N(m-r, c-m-1, k-1) q_{1}^{m+1} p_{1}^{c-m-1} \\
& \times \sum_{w=0}^{n-r-2} \sum_{k-c+m}^{j=m+w+1} N(j-m-w-1, n-c-j+m+1, k-1) q_{2}^{j-m-1} p_{2}^{n-c-j+m+1} \\
& +q_{1}^{c} \sum_{w=0}^{n-1} \sum_{j=c+w}^{k-n+c-2} N(j-c-w, n-j, k-1) q_{2}^{j-c} p_{2}^{n-j} \\
& +q_{2}^{n-c} \sum_{r=0}^{n-1} \sum_{j=n-c+r+1}^{n-1} N(j-n+c-r-1, n-j, k-1) q_{1}^{j-n+c} p_{1}^{n-j},
\end{aligned}
$$

where $\sum_{a}^{b}=0$ if $a>b$, and $N(a, s, k-1)$ is computed using Equation (2), take in consideration that $\left(\begin{array}{l}b \\ d\end{array}\right)=0$ if $b, d<0$ or $d>b$.

\section{Proof}

The state of the component at position $c$ is either working or failed. Conditioning on the state of the component at the change point $c$, we have

$$
\mathrm{R}(k, n, c ; \underline{\mathrm{P}})=p_{1} \mathrm{R}_{w}(k, n, c ; \underline{\mathrm{P}})+q_{1} \mathrm{R}_{F}(k, n, c ; \underline{\mathrm{P}}) .
$$

Now, we try to find $\mathrm{R}_{w}(k, n, c ; \underline{\mathrm{P}})$ and $\mathrm{R}_{F}(k, n, c ; \underline{\mathrm{P}})$. Suppose that we have $j$ failed components in the system, $m$ failures from these $j$ appearing before the change point $\mathrm{c}$, and the remaining $j-m$ failures appearing after $c$.

(i) For computing $\mathrm{R}_{w}(k, n, c ; \underline{\mathrm{P}})$ we argue as follows:

In this case $0 \leq j \leq n-1$, where $0 \leq m \leq c-1$ and $0 \leq j-m \leq n-c$. 


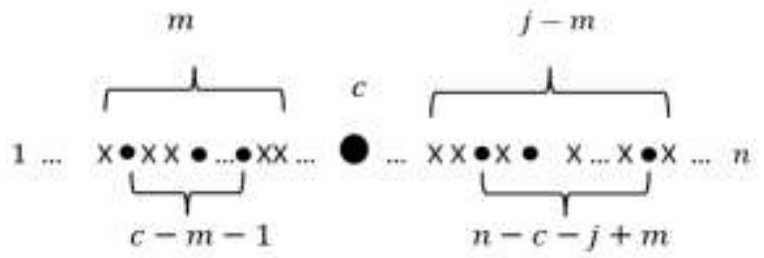

Figure 1: System with $j$ failures and the component at position $c$ is working.

Figure 1 shows a system with $j$ failures, such that $m$ failures and $c-m-1$ working components are before $\mathrm{c}$, and $j-m$ failures and $n-c-j+m$ working components are after c. The component at position $c$ is operating with reliability $p_{1}$, in this case the position of $c$ does not affect the system failure. For the system to operate, we must prevent the appearance of $k$ consecutive failures before and after the change point $c$. The $m$ failures may occupy any position from 1 to $c-1$, with no consecutive $k$ failures, and this can be done by $N(m, c-m, k-1)$ ways. The remaining $j-m$ failures may occupy any position from $c+1$ to $n$ with no consecutive $k$ failures, with $N(j-m, n-c-j+m+1, k-1)$ ways. For each $m$, each arrangement has probability $q_{1}^{m} p_{1}^{c-m-1}$ before the change point $c$, and probability $q_{2}^{j-m} p_{2}^{n-c-j+m}$ after $c$. This means that

$$
\begin{aligned}
\mathrm{R}_{\mathrm{w}}(k, n, c ; \underline{\mathrm{P}}) & =\sum_{\substack{m=0 \\
n-c+m}}^{c-1} N(m, c-m, k-1) q_{1}^{m} p_{1}^{c-m-1} \\
& \times \sum_{j=m} N(j-m, n-c-j+m+1, k-1) q_{2}^{j-m} p_{2}^{n-c-j+m} .
\end{aligned}
$$

(ii) For computing $\mathrm{R}_{F}(k, n, c ; \underline{\mathrm{P}})$, we argue as follows:

The component at position $c$ fails with probability of failure $q_{1}$, in this case the position of $c$ affects the system failure. Then we have the following three cases for the system to operate:

(a) $r$ failures from the $m$ failures before $c$ are directly located before the position $c$, and $w$ failures from the $j-m$ failures after $c$, are directly located after $c$, this situation is depicted by Figure 2, this case for any position of $c$, and $1 \leq j \leq n-2$.

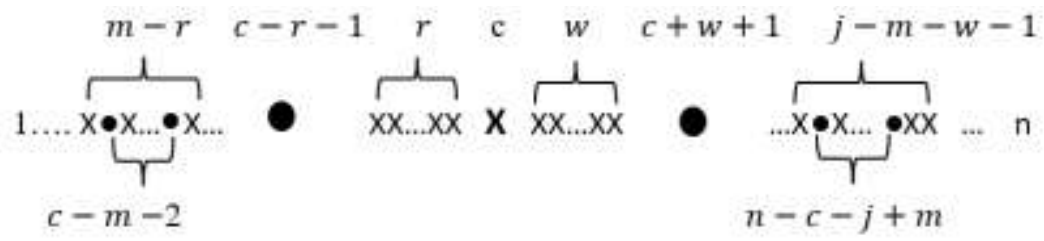

Figure 2: System with $j$ failures and the component at position $c$ is failed.

Figure 2 shows a system with $j$ failures, where $r$ consecutive failures from the $m$ failures are directly located before $c$, and $w$ consecutive failures from the $j-m$ failures are directly located after $c$. The system operates if $r+w+1 \leq k-1$, in this case $0 \leq r \leq$ $k-2$ and $0 \leq w \leq k-r-2$, and at each of positions $c-r-1$ and 
$c+w+1$, we must have a working component. The remaining $m-r$ and $j-m-w-1$ failures can take any positions in the remaining $c-r-2$ and $n-c-w-1$ positions, respectively, without $k$ consecutive failures, and this can be done by $N(m-r, c-m-1, k-1)$ and $N(j-m-w-1, n-c-j+m+1, k-1)$ arrangements, respectively. Each arrangement has probability $q_{1}^{m} p_{1}^{c-m-1}$ before the change point $c$, and $q_{2}^{j-m-1} p_{2}^{n-c-j+m+1}$ after $c$, with $1 \leq j \leq n-2$. Hence this possibility occurs with probability

$$
\begin{aligned}
& \sum_{r=0}^{k-2} \sum_{\substack{m=r \\
k-r-2}}^{c-2} N(m-r, c-m-1, k-1) q_{1}^{m} p_{1}^{c-m-1} \\
& \quad \times \sum_{w=0}^{n-c+m} \sum_{j=m+w+1}^{n} N(j-m-w-1, n-c-j+m+1, k-1) q_{2}^{j-m-1} p_{2}^{n-c-j+m+1} .
\end{aligned}
$$

(b) All components before $c$ are failures, $c<k$.

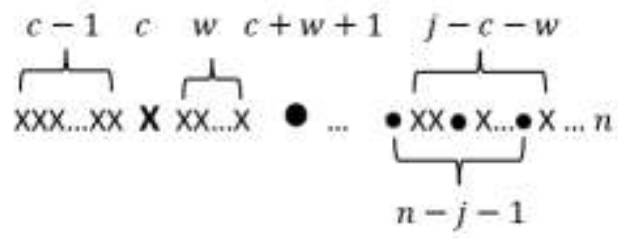

Figure 3: System with $j$ failures and the first $c$ components failed.

Figure 3 shows a system with $j$ failures, the first $c$ components failed, and $w$ consecutive failures occur directly after those $c$ failures. In this case $c+w \leq j \leq n-1$, if $w$ of consecutive failures occur directly after the first $c$ failed components, then the system operates if $c+w \leq k-1$, then $w$ can take values from 0 to $k-c-1$. At position $c+w+1$, we must have a working component. The remaining $j-c-w$ failures can occupy any position in the remaining $n-c-w-1$ positions, without $k$ consecutive failures. There are $N(j-c-w, n-j, k-1)$ arrangements that satisfy the condition. Since for each $w$, each arrangement has probability $q_{1}^{c-1}$ before the change point $c$, and $q_{2}^{j-c} p_{2}^{n-j}$ after this point. Then this possibility occurs with probability

$$
q_{1}^{c-1} \sum_{w=0}^{k-c-1} \sum_{j=c+w}^{n-1} N(j-c-w, n-j, k-1) q_{2}^{j-c} p_{2}^{n-j}
$$

(c) All components after $c$ failed, $c>n-k+1$.

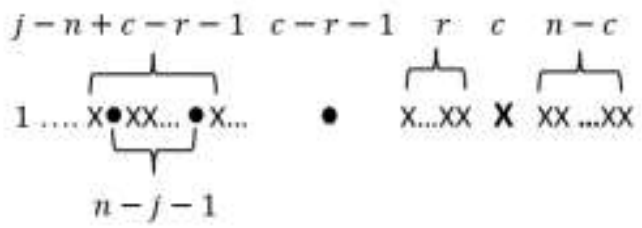

Figure 4: System with $j$ failures and the last $n-c$ components failed. 
Figure 4 shows a system with $j$ failures, the last $n-c$ components fail and $r$ consecutive failures occur directly before position $c$. In this case we have failures at the last $n-c$ positions, position $c$, and $r$ consecutive failures before $c$. In order the system not to fail, we must have $n-c+r+1 \leq k-1$, this means that $0 \leq r \leq k-n+c-2$ and at position $c-r-1$ we must have a working component. The remaining $j-n-r+c-1$ failures can occupy any position in the remaining $c-r-2$ positions, without $k$ consecutive failures, and this can be done by $N(j-n+c-r-1, n-j, k-1)$ arrangement. Since for each $r$, each arrangement has probability $q_{1}^{j-n+c-1} p_{1}^{n-j}$ before the change point $c$, and $q_{2}^{n-c}$ after $c$, with $n-c+r+1 \leq j \leq n-1$. Then the probability of this case is

$$
q_{2}^{n-c} \sum_{r=0}^{k-n+c-2} \sum_{j=n-c+r+1}^{n-1} N(j-n+c-r-1, n-j, k-1) q_{1}^{j-n+c-1} p_{1}^{n-j}
$$

Combining (6), (7), and (8) we get

$$
\begin{aligned}
& \mathrm{R}_{F}(k, n, c ; \underline{\mathrm{P}}) \\
& =\sum_{r=0}^{k-2} \sum_{\substack{r=r \\
k-r-2}}^{c-2} N(m-r, c-m-1, k-1) q_{1}^{m} p_{1}^{c-m-1} \\
& \times \sum_{w=0}^{n-c+m} \sum_{j=m+w+1}^{n-m} N(j-m-w-1, n-c-j+m+1, k \\
& +q_{1}^{c-1} \sum_{w=0}^{-1) q_{2}^{j-m-1} p_{2}^{n-c-j+m+1} \sum_{j=c+w}^{n-1} N(j-c-w, n-j, k-1) q_{2}^{j-c} p_{2}^{n-j}} \\
& +q_{2}^{n-c} \sum_{r=0}^{n-n+c-2} \sum_{j=n-c+r+1}^{n-1} N(j-n+c-r-1, n-j, k-1) q_{1}^{j-n+c-1} p_{1}^{n-j} .
\end{aligned}
$$

Substituting (5), and (9) in (4) the proof is completed.

The following corollary presents the formulas of $\mathrm{R}_{u}(k, n, c ; \underline{\mathrm{P}})$ and $\mathrm{R}_{b}(k, n, c ; \underline{\mathrm{P}})$.

\section{Corollary 1.}

Assuming independent components, for any $k \leq n, 1 \leq c \leq n$ we have

$$
\begin{gathered}
\mathrm{R}_{u}(k, n, c ; \underline{\mathrm{P}})=p_{1} \mathrm{R}(k, n-1, c-1 ; \underline{\mathrm{P}}), \text { and } \\
\mathrm{R}_{b}(k, n, c ; \underline{\mathrm{P}})=p_{1} p_{2} \mathrm{R}(k, n-2, c-1 ; \underline{\mathrm{P}}) .
\end{gathered}
$$

\section{Remark.}

Substituting with $c=n$ and $p_{1}=p, q_{1}=q$ in (3), we obtain the same result as (1) which is the result obtained by Derman et al. (1982) for consecutive k-out-of-n: F system without change point. Similarly substituting with $c=0$ and $p_{2}=p, q_{2}=q$ in (3), we obtain (1). Clearly (1) can be written as

$$
\mathrm{R}(k, n ; p)=\sum_{j=0}^{n-1} a_{n, j} p^{j} q^{n-j}, \text { where } a_{n, j}= \begin{cases}\left(\begin{array}{l}
n \\
j
\end{array}\right) & \text { if } j<k \\
N(j, n-j+1, k-1) & \text { if } j \geq k\end{cases}
$$


Note that if $j=n$ in $(1)$, the term $N(n, 1, k-1)=0$, for any $k$.

\section{Stress-Strength Reliability}

In this section we obtain $\mathrm{R}_{(s: s)}(k, n, c), \mathrm{R}_{(s: s) u}(k, n, c)$, and $\mathrm{R}_{(s: s) b}(k, n, c)$. Suppose that a change in the stress occurs after the component at position $c$. Thus, assume we have two common independent stresses $X_{i}, i=1,2$, with cumulative distribution functions $G_{i}(x), i=1,2$, where the common stress $X_{1}$ is imposed on components 1 to $c$, and the common stress $X_{2}$ is imposed on components $c+1$ to $n$. The strengths $Y_{i}, i=1, \ldots, n$ of the components are independent and identically distributed, having cumulative distribution function $F(x)$. The stress-strength reliability of $L / k / n / c$ is given by the following theorem.

\section{Theorem 2.}

Let $G_{i}(x), i=1,2$ denote the cumulative distribution functions of stresses $X_{i}, i=1,2$, and $F(x)$ denote the cumulative distribution function of strengths $Y_{i}, i=1, \ldots, n$. Assume that $X_{1}$ and $X_{2}$ are independent, and $Y_{i}^{\prime} s$ are independent and identically distributed. The stress-strength reliability of $L / k / n / c$, for any $k \leq n$, and $1 \leq c \leq n$, is given by

$$
\begin{aligned}
& \mathrm{R}_{(s: s)}(k, n, c) \\
= & \sum_{m=0}^{c-1} N(m, c-m, k-1) I_{m, c-m}^{1} \\
& \times \sum_{j=m}^{n-c+m} N(j-m, n-c-j+m+1, k-1) I_{j-m, n-c-j+m}^{2} \\
+ & \sum_{r=0}^{k-2} \sum_{k=r}^{c-2} N(m-r, c-m-1, k-1) I_{m+1, c-m-1}^{1} \\
& \sum_{w=0}^{n-r-2} \sum_{j-c+m}^{j=m+w+1} N(j-m-w-1, n-c-j+m+1, k-1) I_{j-m-1, n-c-j+m+1}^{2} \\
+ & I_{c, 0}^{1} \sum_{w=0}^{n-1} \sum_{k=c+w}^{j-c} N(j-c-w, n-j, k-1) I_{j-c, n-j}^{2} \\
+ & I_{n-c, 0}^{2} \sum_{r=0}^{n-1} \sum_{j=n-c+r+1}^{n+1} N(j-n+c-r-1, n-j, k-1) I_{j-n+c, n-j}^{1},
\end{aligned}
$$

where

$$
I_{a, b}^{i}=\int_{0}^{\infty}[F(x)]^{a}[1-F(x)]^{b} d G_{i}(x), \quad i=1,2 .
$$

\section{Proof}

We can easily show that (12) could be obtained by substituting $q_{i}^{s} p_{i}^{h-s}$ in (3) by $\int_{0}^{\infty}[F(x)]^{s}[1-F(x)]^{h-s} d G_{i}(x), i=1,2$, where 


\section{Corollary 2}

$$
h=\left\{\begin{array}{cc}
c & \text { with } \quad s=m, m+1, c, \text { or } j-n+c \text { if } i=1 \\
n-c & \text { with } s=j-m, j-m-1, j-c \text {, or } n-c \text { if } i=2
\end{array} .\right.
$$

Let $X_{1}$ and $X_{2}$ be two common stresses with cumulative distribution functions $G_{1}(x)$ and $G_{2}(x)$, imposed on components 1 to $c$ and components $c+1$ to $n$, respectively. Assume that $X_{1}$ and $X_{2}$ are independent. Let $Y_{i}, i=1, \ldots, n$ denote the strengths of components $1, \ldots, n$. Assume that $Y_{i}^{\prime} s$ are independent and identically distributed. For any $k \leq n$, and $1 \leq c \leq n$, the stress-strength reliability of a unipolarrelayed and bipolar-relayed $L / k / n / c$ system, is given respectively by

$$
\begin{aligned}
& \mathrm{R}_{(s: s) u}(k, n, c) \\
= & \sum_{m=0}^{c-2} N(m, c-m-1, k-1) I_{m, c-m}^{1} \\
& \times \sum_{j=m}^{n-c+m} N(j-m, n-c-j+m+1, k-1) I_{j-m, n-c-j+m}^{2} \\
& +\sum_{r=0}^{k-2} \sum_{m=r}^{c-3} N(m-r, c-m-2, k-1) I_{m+1, c-m-1}^{1} \\
& \times \sum_{w=0}^{n-r-2} \sum_{\substack{n-c+m \\
k-c}}^{n+w+1} N(j-m-w-1, n-c-j+m+1, k-1) I_{j-m-1, n-c-j+m+1}^{2} \\
+ & I_{c-1,1}^{1} \sum_{w=0}^{n-2} \sum_{k-n+w-1} N(j-c-w+1, n-j-1, k-1) I_{j-c+1, n-j-1}^{2} \\
+ & I_{n-c, 0}^{2} \sum_{r=0}^{n-2} \sum_{j=n-c+r+1} N(j-n+c-r-1, n-j-1, k-1) I_{j-n+c, n-j}^{1}
\end{aligned}
$$

and

$$
\begin{aligned}
& \mathrm{R}_{(s: s) b}(k, n, c) \\
& =\sum_{\substack{m=0 \\
n-c+m-1}} N(m, c-m-1, k-1) I_{m, c-m}^{1} \\
& \times \sum_{j=m}^{k-2} N(j-m, n-c-j+m, k-1) I_{j-m, n-c-j+m}^{2} \\
& +\sum_{r=0}^{c-3} \sum_{m=r}^{c-3} N(m-r, c-m-2, k-1) I_{m+1, c-m-1}^{1} \\
& \times \sum_{w=0}^{k-r-2} \sum_{\substack{n=m+m-1 \\
k-c}}^{n-w+1} N(j-m-w-1, n-c-j+m, k-1) I_{j-m-1, n-c-j+m+1}^{2} \\
& +I_{c-1,1}^{1} \sum_{w=0}^{n-3} \sum_{j=c+w-1} N(j-c-w+1, n-j-2, k-1) I_{j-c+1, n-j-1}^{2}
\end{aligned}
$$




$$
+I_{n-c-1,1}^{2} \sum_{r=0}^{k-n+c-1} \sum_{j=n-c+r}^{n-3} N(j-n+c-r, n-j-2, k-1) I_{j-n+c+1, n-j-1}^{1} .
$$

\section{The Exact Stress-Strength Reliability Formulas for Some Special Distributions}

The exact reliability formulas of $\mathrm{R}_{(s: s)}(k, n, c), \mathrm{R}_{(s: s) u}(k, n, c)$, and $\mathrm{R}_{(s: s) b}(k, n, c)$ are obtained in two cases: case I, when the strength and the stresses have the same form of distributions. As an application of this case, we consider the general exponential form. Case II, when the strength and the stresses have different forms of distributions, and as application, we consider the generalized Lindley distribution for strength, while the stresses have a negative exponential distribution.

\section{Case I}

Al-Hussaini (1999) has proposed that survival function $\bar{F}_{X}$ of any continuous random variable can be expressed by the form $\bar{F}_{X}(x)=e^{-\lambda(x, \theta)}$, where $\theta$ could be a vector parameter, $\lambda(x, \theta)$ is a continuous, monotone increasing, and differentiable function such that $\lambda(x, \theta) \rightarrow 0$ as $x \rightarrow-\infty$ and $\lambda(x, \theta) \rightarrow \infty$ as $x \rightarrow \infty$. Here we shall consider $\lambda(x, \theta)=\delta g(x, \vartheta), \delta>0, g(x, \vartheta)$ does not contain $\delta$, and $g(x, \vartheta)$ is a continuous, monotone increasing, and differentiable function such that $g(x, \vartheta) \rightarrow 0$ as $x \rightarrow-\infty$ and $g(x, \vartheta) \rightarrow \infty$ as $x \rightarrow \infty$. We consider distributions with cumulative distribution function, given by

$$
H(x)=1-e^{-\delta g(x, \vartheta)}, \delta>0 .
$$

The distribution with form (16) is called general exponential form distribution, see Mokhlis et al. (2017). Many distributions have cumulative distribution functions satisfying the form in (16). Table 1 presents some examples of these distributions.

Table 1: The cumulative distribution function of some distributions that belongs to the general exponential form

\begin{tabular}{ccc}
\hline Distribution & $H(x)$ & $g(x, \vartheta)$ \\
\hline Negative Exponential & $1-e^{-\delta x}$ & $x$ \\
Weibull & $1-e^{-\delta x^{\vartheta}}$ & $x^{\vartheta}$ \\
Pareto & $1-\left(\frac{\vartheta}{x}\right)^{\delta}$ & $\ln \left(\frac{x}{\vartheta}\right)$ \\
Kumaraswamy & $1-\left(1-x^{\vartheta}\right)^{\delta}$ & $\ln \left(x^{\vartheta}-1\right)$ \\
\hline
\end{tabular}

Assume that the cumulative distribution functions of the stresses $X_{1}$ and $X_{2}$, and strength $Y$ are given by

$G_{i}(x)=1-e^{-\lambda_{i} g(x, \vartheta)} ; \lambda_{i}>0, i=1,2$, and

$F(x)=1-e^{-\delta g(x, \vartheta)}, \delta>0$.

By substituting the forms of $G_{i}(x), i=1,2$ and $F(x)$ given by (17) and (18) respectively, in (13), we get 


$$
\begin{aligned}
I_{a, b}^{i} & =\int_{0}^{\infty}\left[1-e^{-\delta g(x, \vartheta)}\right]^{a}\left[e^{-\delta g(x, \vartheta)}\right]^{b} \mathrm{~d}\left(1-e^{-\lambda_{i} g(x, \vartheta)}\right) \\
& =\sum_{l=0}^{a}\left(\begin{array}{l}
a \\
l
\end{array}\right)(-1)^{l} \frac{\lambda_{i}}{(l+b) \delta+\lambda_{i}}=I_{a, b}^{i}\left(\delta, \lambda_{i}\right), i=1,2,
\end{aligned}
$$

which does not contain the parameter $\vartheta$. Substituting (19) in (12), (14), and (15), we obtain exact stress-strength reliability formulas for $\mathrm{R}_{(s: s)}(k, n, c), \mathrm{R}_{(s: s) u}(k, n, c)$, and $\mathrm{R}_{(s: s) b}(k, n, c)$. Since (19) does not contain the parameter $\vartheta$, the stress-strength reliability formulas also do not contain the parameter $\vartheta$.

\section{Case II}

Suppose that the distributions of stresses $X_{1}$ and $X_{2}$ are negative exponential, with cumulative distribution functions are given by

$$
G_{i}(x)=1-e^{-\lambda_{i} x}, i=1,2 .
$$

Let the distribution of the strength $Y$ is a generalized Lindley distribution, with probability distribution and cumulative distribution function given respectively by

$$
\begin{gathered}
f(y)=\frac{1}{1+\theta}\left[\frac{\theta^{\alpha+1} y^{\alpha-1}}{\Gamma(\alpha)}+\frac{\theta^{\beta} y^{\beta-1}}{\Gamma(\beta)}\right] e^{-\theta y}, \text { and } \\
F(x)=p(Y \leq x)=\frac{1}{1+\theta}\left[\frac{\theta \gamma(\alpha, \theta x)}{\Gamma(\alpha)}+\frac{\gamma(\beta, \theta x)}{\Gamma(\beta)}\right], \theta, \alpha, \beta>0,
\end{gathered}
$$

where $\gamma(s, v x)$ is a lower incomplete gamma. We can easily see that $f(y)$ is a mixture of two gamma distributions, with parameters $(\theta, \alpha)$ and $(\theta, \beta)$ with probabilities $\frac{\theta}{1+\theta}$ and $\frac{1}{1+\theta}$ respectively. Substituting $G_{i}(x), \mathrm{i}=1,2$ and $F(x)$ given by (20) and (21) respectively in (13), we have

$$
\begin{aligned}
I_{a, b}^{i}= & \int_{0}^{\infty}\left[\frac{1}{1+\theta}\left[\frac{\theta \gamma(\alpha, \theta x)}{\Gamma(\alpha)}+\frac{\gamma(\beta, \theta x)}{\Gamma(\beta)}\right]\right]^{a}\left[1-\frac{1}{1+\theta}\left[\frac{\theta \gamma(\alpha, \theta x)}{\Gamma(\alpha)}+\frac{\gamma(\beta, \theta x)}{\Gamma(\beta)}\right]\right]^{b} \mathrm{~d}\left(1-e^{-\lambda_{i} x}\right) \\
& =I_{a, b}^{i}\left(\theta, \alpha, \beta, \lambda_{i}\right), i=1,2 .
\end{aligned}
$$

The above integral can be obtained numerically, and hence the expression for $\mathrm{R}_{(s: s)}(k, n, c), \mathrm{R}_{(s: s) u}(k, n, c)$, and $\mathrm{R}_{(s: s) b}(k, n, c)$.

\section{Estimation of The Stress-Strength Reliability}

\section{Case I}

As an application of the general exponential form, we take for simplicity $g(x, \vartheta)=x$. The maximum likelihood estimator of $\mathrm{R}_{(s: s)}(k, n, c), \mathrm{R}_{(s: s) u}(k, n, c)$, and $\mathrm{R}_{(s: s) b}(k, n, c)$, can be obtained by replacing the parameters in (19) by their corresponding maximum likelihood estimators. For obtaining the maximum likelihood estimator of the parameters, let $X_{1}, X_{2}, \ldots, X_{n_{i}} ; i=1,2$, and $Y_{1}, Y_{2}, \ldots, Y_{n_{3}}$ be samples of size $n_{i}, i=1,2$, and $n_{3}$ from stresses and strength distributions. Clearly the maximum likelihood estimators of the parameters $\lambda_{i}, \delta$ are given by 
Hence

$$
\begin{gathered}
\hat{\lambda}_{i}=\frac{n_{i}}{\sum_{r=0}^{n_{i}} X_{r}}, i=1,2, \text { and } \\
\hat{\delta}=\frac{n_{3}}{\sum_{r=0}^{n_{3}} Y_{r}} .
\end{gathered}
$$

$$
\hat{I}_{a, b}^{i}\left(\hat{\delta}, \hat{\lambda}_{i}\right)=\sum_{l=0}^{a}\left(\begin{array}{l}
a \\
l
\end{array}\right)(-1)^{l} \frac{n_{i} \sum_{r=0}^{n_{3}} Y_{r}}{n_{i} \sum_{r=0}^{n_{3}} Y_{r}+(l+b) n_{3} \sum_{r=0}^{n_{i}} X_{r}}, i=1,2 .
$$

Replacing $I_{a, b}^{i}, i=1,2$ in Equations (12), (14), and (15) by $\hat{I}_{a, b}^{i}\left(\hat{\delta}, \hat{\lambda}_{i}\right)$ given by (25), we get the maximum likelihood estimators $\widehat{\mathrm{R}}_{(s: s)}(k, n, c), \widehat{\mathrm{R}}_{(s: s) u}(k, n, c)$, and $\widehat{\mathrm{R}}_{(s: s) b}(k, n, c)$ of $\mathrm{R}_{(s: s)}(k, n, c), \mathrm{R}_{(s: s) u}(k, n, c)$, and $\mathrm{R}_{(s: s) b}(k, n, c)$, respectively.

\section{Case II}

The maximum likelihood estimators of the parameters $\lambda_{i}, i=1,2$ are given by (23). Since the generalized Lindley distribution in (21) is a mixture of two gamma distributions, we use the EM algorithm for obtaining the estimators $\hat{\theta}, \hat{\alpha}$, and $\hat{\beta}$ of $\theta, \alpha$, and $\beta$ respectively. Estimators of $\mathrm{R}_{(s: s)}(k, n, c), \mathrm{R}_{(s: s) u}(k, n, c)$, and $\mathrm{R}_{(s: s) b}(k, n, c)$ are obtained by substituting the parameters with their corresponding estimators $\hat{\theta}, \hat{\alpha}$, and $\hat{\beta}$.

\section{Numerical Illustration}

Tables (2), (3), and (4) present the exact stress-strength reliabilities for case I (same general exponential form distributions), with $g(x, \vartheta)=x$, while Tables (5), (6), and (7) illustrate the reliabilities for case II (different forms of distributions), showing the effect of position of the change point $c$, the value of $k$ with respect to $n$, and different parameters, on reliabilities. Using R-programming we compute $\mathrm{R}_{(s: s)}(k, n, c)$, $\mathrm{R}_{(s: s) u}(k, n, c)$, and $\mathrm{R}_{(s: s) b}(k, n, c)$ with $n=6, k=(2,3$, and 4$)$, and different values of the parameters of the distributions of the stresses and strength, with different values of $c=(2$, and 4)i.e $(c \leq k$, and $c>k)$. In Table (8) we present the maximum likelihood estimator of the reliabilities, obtained in Section 5. The estimators $\widehat{\mathrm{R}}_{(s: s)}(k, n, c)$,

$\widehat{\mathrm{R}}_{(s: s) u}(k, n, c)$, and $\widehat{\mathrm{R}}_{(s: s) b}(k, n, c)$, that appear in these table are the average of 1000 repetitions, and we take $n_{i}=20,50,100, i=1,2,3$. The mean square error of the estimators is also calculated to indicate the accuracy of the estimators.

It is clear from Tables (2) to (7) that all reliabilities increase as $k$ increases for all cases of $c$, and for the different values of the parameters. The position of change point $c$ also influence the reliability according to the rate of stress before or after this point and number of components under this stress. We also see the effect of the strength and stresses parameters, on the reliability of the system in both cases I, and II. For case I, for the strength parameters, we can see that the increase of $\delta$ causes decrease in the reliability, while for stresses parameters, as $\lambda_{1}$ or $\lambda_{2}$ increase the reliability increases. From Tables $(2-4)$, we see that the highest value of the reliability is attained for the largest values of $\lambda_{1}$ and $\lambda_{2}$, and the smallest value of $\delta$. Also, for generalized Lindley strength, we see from Tables $(5-7)$ as $\theta$ increases the reliability decreases, but as $\alpha$ or $\beta$ increase the reliability increases. Also, we have the highest value of the reliability 
attained when the values of $\alpha, \beta$, and $\lambda_{i}(i=1,2)$ are large and $\theta$ is small. From Table (8), we see that bias and the mean square errors are small. The mean square error in all cases decreases by increasing the sample size. However, the estimators are satisfactory. Also, we see that $\operatorname{MSE}\left(\widehat{\mathrm{R}}_{(s: s)}(k, n, c)\right)<\operatorname{MSE}\left(\widehat{\mathrm{R}}_{(s: s) u}(k, n, c)\right)<$ $\operatorname{MSE}\left(\widehat{\mathrm{R}}_{(s: s) b}(k, n, c)\right)$ for all cases.

Table 2: Exact reliabilities for case I, with $k=2$

\begin{tabular}{ccccccc}
\hline$c$ & $\delta$ & $\lambda_{1}$ & $\lambda_{2}$ & $\mathrm{R}_{(s: s)}(k, n, c)$ & $\mathrm{R}_{(s: s) u}(k, n, c)$ & $\mathrm{R}_{(s: s) b}(k, n, c)$ \\
\hline \multirow{2}{*}{2} & 4 & 8 & 6 & 0.494228 & 0.3910534 & 0.3102453 \\
& 4 & 6 & 8 & 0.5171429 & 0.3971429 & 0.3228571 \\
& 1 & 8 & 6 & 0.8874074 & 0.8059259 & 0.7185185 \\
& 4 & 3 & 6 & 0.3415416 & 0.2450666 & 0.1948052 \\
& 4 & 8 & 2 & 0.2481481 & 0.1957672 & 0.1449735 \\
\hline \multirow{4}{*}{4} & 4 & 8 & 6 & 0.5171429 & 0.42 & 0.3228571 \\
& 4 & 6 & 8 & 0.494228 & 0.3917749 & 0.3102453 \\
& 1 & 8 & 6 & 0.9025974 & 0.8220779 & 0.7298701 \\
& 4 & 3 & 6 & 0.3048804 & 0.2304033 & 0.176432 \\
& 4 & 8 & 2 & 0.3088889 & 0.2511111 & 0.1755556 \\
\hline
\end{tabular}

Table 3: Exact reliabilities for case I, with $k=3$

\begin{tabular}{ccccccc}
\hline$c$ & $\delta$ & $\lambda_{1}$ & $\lambda_{2}$ & $\mathrm{R}_{(s: s)}(k, n, c)$ & $\mathrm{R}_{(s: s) u}(k, n, c)$ & $\mathrm{R}_{(s: s) b}(k, n, c)$ \\
\hline \multirow{3}{*}{2} & 4 & 8 & 6 & 0.7520924 & 0.5246753 & 0.3665224 \\
& 4 & 6 & 8 & 0.8019048 & 0.5085714 & 0.3742857 \\
& 1 & 8 & 6 & 0.9759259 & 0.8698413 & 0.7540741 \\
& 4 & 3 & 6 & 0.6912801 & 0.3335807 & 0.2339349 \\
& 4 & 8 & 2 & 0.4444444 & 0.3153439 & 0.184127 \\
\hline \multirow{4}{*}{4} & 4 & 8 & 6 & 0.8019048 & 0.5971429 & 0.3742857 \\
& 4 & 6 & 8 & 0.7520924 & 0.5310245 & 0.3665224 \\
& 1 & 8 & 6 & 0.9844156 & 0.8805195 & 0.7571429 \\
& 4 & 3 & 6 & 0.5461693 & 0.3472044 & 0.2203964 \\
& 4 & 8 & 2 & 0.7333333 & 0.5577778 & 0.2066667 \\
\hline
\end{tabular}

Table 4: Exact reliabilities for case I, with $k=4$

\begin{tabular}{ccccccc}
\hline$c$ & $\delta$ & $\lambda_{1}$ & $\lambda_{2}$ & $\mathrm{R}_{(s: s)}(k, n, c)$ & $\mathrm{R}_{(s: s) u}(k, n, c)$ & $\mathrm{R}_{(s: s) b}(k, n, c)$ \\
\hline \multirow{3}{*}{2} & 4 & 8 & 6 & 0.8626263 & 0.5858586 & 0.3930736 \\
& 4 & 6 & 8 & 0.9047619 & 0.5542857 & 0.3942857 \\
& 1 & 8 & 6 & 0.9939153 & 0.8840212 & 0.7612698 \\
& 4 & 3 & 6 & 0.8337662 & 0.3745994 & 0.2506662 \\
& 4 & 8 & 2 & 0.5640212 & 0.3873016 & 0.2137566 \\
\hline \multirow{3}{*}{4} & 4 & 8 & 6 & 0.9047619 & 0.6457143 & 0.3942857 \\
& 4 & 6 & 8 & 0.8626263 & 0.5803752 & 0.3930736 \\
& 1 & 8 & 6 & 0.9968254 & 0.8878788 & 0.7614719
\end{tabular}




\begin{tabular}{llllll}
4 & 3 & 6 & 0.6800312 & 0.4005742 & 0.2481437 \\
4 & 8 & 2 & 0.8755556 & 0.6266667 & 0.2177778 \\
\hline
\end{tabular}

Table 5: Exact reliabilities for case II, with $\boldsymbol{k}=\mathbf{2}$

\begin{tabular}{ccccccccc}
\hline$c$ & $\theta$ & $\alpha$ & $\beta$ & $\lambda_{1}$ & $\lambda_{2}$ & $\mathrm{R}_{(s: s)}(k, n, c)$ & $\mathrm{R}_{(s: s) u}(k, n, c)$ & $\mathrm{R}_{(s: s) b}(k, n, c)$ \\
\hline & 1 & 3 & 2 & 8 & 6 & 0.9978886 & 0.9875958 & 0.9804053 \\
2 & 1 & 3 & 2 & 6 & 8 & 0.9986011 & 0.9912793 & 0.9810692 \\
& 7 & 3 & 2 & 8 & 6 & 0.8136749 & 0.7518598 & 0.6855084 \\
& 1 & 5 & 2 & 8 & 6 & 0.9985046 & 0.992505 & 0.9828109 \\
& 1 & 3 & $1 / 2$ & 8 & 6 & 0.8411834 & 0.7229847 & 0.6116767 \\
\hline \multirow{3}{*}{4} & 1 & 3 & 2 & 8 & 6 & 0.9986011 & 0.9920017 & 0.9810692 \\
& 1 & 3 & 2 & 6 & 8 & 0.9978886 & 0.9869422 & 0.9804053 \\
& 7 & 3 & 2 & 8 & 6 & 0.8347424 & 0.7774817 & 0.6998564 \\
& 1 & 5 & 2 & 8 & 6 & 0.9989932 & 0.9929991 & 0.9832741 \\
& 1 & 3 & $1 / 2$ & 8 & 6 & 0.8535313 & 0.7354143 & 0.6207246 \\
\hline
\end{tabular}

Table 6: Exact reliabilities for case II, with $k=3$

\begin{tabular}{ccccccccc}
\hline$c$ & $\theta$ & $\alpha$ & $\beta$ & $\lambda_{1}$ & $\lambda_{2}$ & $\mathrm{R}_{(s: s)}(k, n, c)$ & $\mathrm{R}_{(s: s) u}(k, n, c)$ & $\mathrm{R}_{(s: s) b}(k, n, c)$ \\
\hline & 1 & 3 & 2 & 8 & 6 & 0.9998605 & 0.9883034 & 0.9814962 \\
2 & 1 & 3 & 2 & 6 & 8 & 0.9999604 & 0.9930054 & 0.981542 \\
& 7 & 3 & 2 & 8 & 6 & 0.9266386 & 0.8234927 & 0.716885 \\
& 1 & 5 & 2 & 8 & 6 & 0.9999275 & 0.9937484 & 0.9836141 \\
& 1 & 3 & $1 / 2$ & 8 & 6 & 0.9692297 & 0.8114427 & 0.6638775 \\
\hline \multirow{3}{*}{4} & 1 & 3 & 2 & 8 & 6 & 0.9999604 & 0.9931194 & 0.981542 \\
& 1 & 3 & 2 & 6 & 8 & 0.9998605 & 0.9882719 & 0.9814962 \\
& 7 & 3 & 2 & 8 & 6 & 0.9582906 & 0.8689741 & 0.7228816 \\
& 1 & 5 & 2 & 8 & 6 & 0.9999771 & 0.9938056 & 0.9836378 \\
& 1 & 3 & $1 / 2$ & 8 & 6 & 0.9748258 & 0.8179912 & 0.666214 \\
\hline
\end{tabular}

Table 7: Exact reliabilities for case II, with $k=\mathbf{4}$

\begin{tabular}{ccccccccc}
\hline$c$ & $\theta$ & $\alpha$ & $\beta$ & $\lambda_{1}$ & $\lambda_{2}$ & $\mathrm{R}_{(s: s)}(k, n, c)$ & $\mathrm{R}_{(s: s) u}(k, n, c)$ & $\mathrm{R}_{(s: s) b}(k, n, c)$ \\
\hline & 1 & 3 & 2 & 8 & 6 & 0.999987 & 0.988336 & 0.9815591 \\
2 & 1 & 3 & 2 & 6 & 8 & 0.9999977 & 0.9931286 & 0.9815593 \\
& 7 & 3 & 2 & 8 & 6 & 0.9606657 & 0.8507304 & 0.730014 \\
& 1 & 5 & 2 & 8 & 6 & 0.9999954 & 0.9938142 & 0.9836479 \\
& 1 & 3 & $1 / 2$ & 8 & 6 & 0.9939743 & 0.8286248 & 0.6729649 \\
\hline \multirow{3}{*}{4} & 1 & 3 & 2 & 8 & 6 & 0.9999977 & 0.993141 & 0.9815593 \\
& 1 & 3 & 2 & 6 & 8 & 0.999987 & 0.9883376 & 0.9815591 \\
& 7 & 3 & 2 & 8 & 6 & 0.9817882 & 0.881405 & 0.7302931 \\
& 1 & 5 & 2 & 8 & 6 & 0.999999 & 0.9938185 & 0.983648 \\
& 1 & 3 & $1 / 2$ & 8 & 6 & 0.9955297 & 0.8305905 & 0.6731956 \\
\hline
\end{tabular}


Table 8: Estimated reliabilities

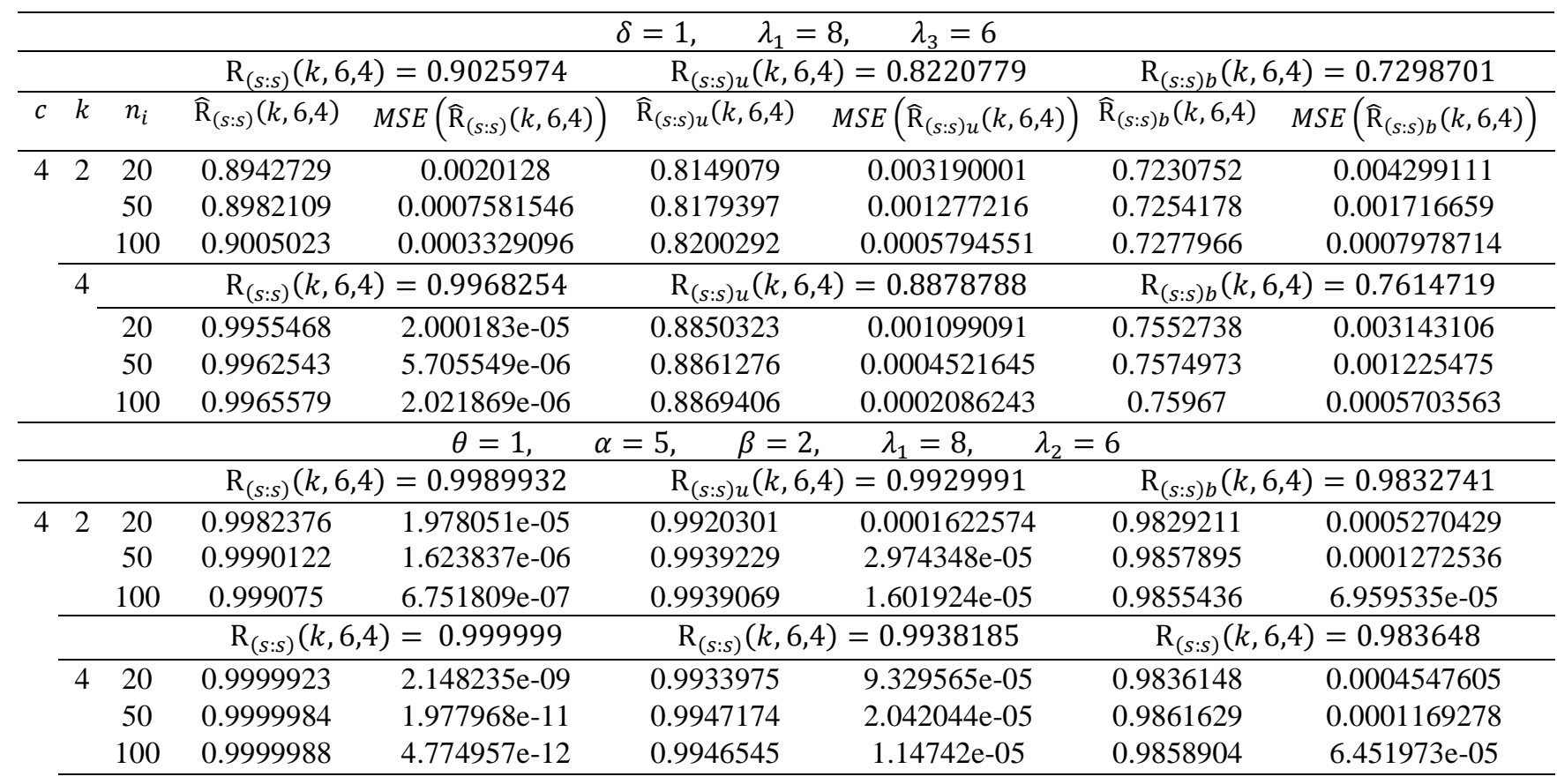

\section{Conclusions}

In this paper explicit expressions for $\mathrm{R}(k, n, c ; \underline{\mathrm{P}}), \mathrm{R}_{u}(k, n, c ; \underline{\mathrm{P}})$, and $\mathrm{R}_{b}(k, n, c ; \underline{\mathrm{P}})$ are obtained conditioning on the state of the change point at position $c$ (working or failed). Then consequently, $\mathrm{R}_{(s: s)}(k, n, c), \mathrm{R}_{(s: s) u}(k, n, c)$, and $\mathrm{R}_{(s: s) b}(k, n, c)$ are presented when the change in the components reliabilities is due to change in the stress. This means that the components from 1 to $c$ are subjected to a common stress, $X_{1}$, while the components from $c+1$ to $n$ are subjected to a different common stress, $X_{2}$. The strengths of all components 1 to $n$ are independent and identical. The stress-strength reliabilities of linear consecutive k-out-of-n: F system, unipolar-relayed and bipolar-relayed linear consecutive k-out-of-n: $\mathrm{F}$ systems are obtained for any distribution of stresses $X_{i}, i=1,2$, and strength $Y$. As application, two cases are discussed: Case I and Case II. In Case I, the stresses and strength are assumed to have the same form of distributions (as an example, general exponential form, in (16)). Exact formulas are obtained in this case, showing that the forms of the stress-strength reliabilities for all systems do not involve the parameter $\vartheta$. In Case II, the stresses and strength are assumed to have different forms of distributions (as an example, negative exponential distribution for stresses and generalized Lindley distribution for strength). Numerical illustrations are applied through simulation studies for both cases, to detect the effect of position of the change point $c$, the value of $k$ with respect to $n$, and different distributions parameters, on the stress strength reliabilities. For both Cases I and II, the simulation studies showed that $\mathrm{R}_{(s: s)}(k, n, c)$, $\mathrm{R}_{(s: s) u}(k, n, c)$, and $\mathrm{R}_{(s: s) b}(k, n, c)$ increase as $k$ increases for any value of $c$, and for the different values of the parameters. It is also shown that the position of change point $c$ influences the reliability, depending on the rate of stress before or after this point and the 
number of components under that stress. Also, the stress-strength reliability of all systems is sensitive to the distributions parameters involved in its form. The maximum likelihood estimators $\widehat{\mathrm{R}}_{(s: s)}(k, n, c), \quad \widehat{\mathrm{R}}_{(s: s) u}(k, n, c) \quad$ and $\quad \widehat{\mathrm{R}}_{(s: s) b}(k, n, c) \quad$ of $\mathrm{R}_{(s: s)}(k, n, c)$, $\mathrm{R}_{(s: s) u}(k, n, c)$ and $\mathrm{R}_{(s: s) b}(k, n, c)$, are their mean square errors are also calculated to indicate the accuracy of the estimation.

\section{References}

1. Akici, F. (2010). Reliability Analysis of Consecutive-k Systems in a StressStrength Setup. (Master's thesis, Graduate School of Natural and Applied Sciences).

2. Al-Hussaini, E. K. (1999). Predicting observables from a general class of distributions. Journal of Statistical Planning and Inference, 79, 79-91.

3. Birnbaum, Z. (1956). On a use of the Mann-Whitney statistic. Proceedings of the third Berkeley symposium on mathematical statistics and probability. University of California Press Berkeley, 13-17.

4. Birnbaum, Z. and Mccarty, R. (1958). A Distribution-Free Upper Confidence Bound for $\mathrm{P}\{\mathrm{Y}<\mathrm{X}\}$, Based on Independent Samples of $\mathrm{X}$ and $\mathrm{Y}$. The Annals of Mathematical Statistics, 558-562.

5. Chao, M., Fu, J. and Koutras, M. (1995). Survey of reliability studies of consecutive-k-out-of-n: F and related systems. IEEE Transactions on Reliability, 44, 120-127.

6. Chiang, D. T. and Niu, S. C. (1981). Reliability of consecutive-k-out-of-n: F system. IEEE Transactions on Reliability, 30, 87-89.

7. Derman, C., Lieberman, G. J. and Ross, M. S. (1982). On the consecutive-k-of-n: F system. IEEE Transactions on Reliability, 31, 57-63.

8. Elbatal, I., Merovci, F. and Elgarhy, M. (2013). A new generalized Lindley distribution. Mathematical theory and Modeling.

9. Eryllmaz, S. (2008). Consecutive k-out-of-n: G system in stress-strength setup. Communications in Statistics Simulation and Computation, 37, 579-589.

10. Ge, G. and Wang, L. (1990). Exact reliability formula for consecutive-k-out-of-n: F systems with homogeneous Markov dependence. IEEE Transactions on Reliability, 39, 600-602.

11. Gökdere, G., Gürcan, M. and Kılıç, M. B. (2016). A new method for computing the reliability of consecutive k-out-of-n: F systems. Open Physics, 14, 166-170.

12. Hwang, F. (1982). Fast solutions for consecutive-k-out-of-n: F system. IEEE Transactions on Reliability, 31, 447-448.

13. Hwang, F. (1988). Relayed consecutive-k-out-of-n: F lines. IEEE Transactions on Reliability, 37, 512-514.

14. Johnson, R. A. (1988). Stress-strength models for reliability. Handbook of statistics, 7, 27-54. 
15. Jung, K. H. and Kim, H. (1993). Linear consecutive-k-out-of-n: F system reliability with common-mode forced outages. Reliability Engineering \& System Safety, 41, 49-55.

16. Kossow, A. and Preuss, W. (1989). Reliability of consecutive-k-out-of-n: F systems with nonidentical component reliabilities. IEEE Transactions on Reliability, 38, 229-233.

17. Lambiris, M. and Papastavridis, S. (1985). Exact reliability formulas for linear \& circular consecutive-k-out-of-n: F systems. IEEE Transactions on Reliability, 34, 124-126.

18. Mokhlis, N. A. (2001). consecutive k-out-of-n systems. In Advances, in Reliability, (N.Balakrishnan \& C.R.Rao, eds.). Elsevier, New York, 237-280".

19. Mokhlis, N. A., Ibrahim, E. J and Gharieb, D. M. (2017). Stress-strength reliability with general form distributions. Communications in Statistics-Theory and Methods, 46, 1230-1246.

20. Zhao, X., Guo, X. and Wang, X. (2018). Reliability and maintenance policies for a two-stage shock model with self-healing mechanism. Reliability Engineering \& System Safety, 172, 185-194. 